\title{
Quantifying Improvement Following CNS Transplantation in Hemiparkinsonian Monkeys Using Operant Behavioral Tasks
}

\author{
R.A.E. Bakay, R.L. Watts, L.D. Byrd and A. Mandir \\ Yerkes Regional Primate Research Center, Emory University, Atlanta, Georgia 30322, USA
}

\section{INTRODUCTION}

One of the difficulties in evaluating the success of CNS transplantation techniques is obtaining quantitative measures of disability characteristic of the parkinsonian model. Our experience with MPTP parkinsonian monkeys suggests that rotational behavior is not a completely satisfactory index of disability. Consequently, we have investigated conditioning techniques for characterizing and quantifying behavioral performance in parkinsonian monkeys.

\section{METHODS}

Three experimental groups were studied: (1) adrenal medullary/sural nerve cografts $(n=6)$; (2) sham surgical controls $(n=4)$, and (3) nonsurgical controls $(n=4)$. Behavioral assessment of motor activity was evaluated by clinical examination, drug-induced rotational analysis, and performance on a learned visual-motor forelimb task. Each monkey was tested before administration of MPTP, after stabilization of the Parkinson-like state (2-4 months post-MPTP), and postoperatively, each monkey thus serving as its own control.

One group $(n=6)$ was trained to perform a discrete-trial operant task requiring the simultaneous depression of two levers on a response panel. Once both levers were depressed, lights above either the right or left lever were darkened to indicate which hand to use in touching an illuminated area on a touch-sensitive screen for a reward. Testing measured separate hand functions in response time (RT), movement time (MT), and precision of responses.

Another group $(n=8)$ was tested on a ballistic wrist flexion/extension task. A potentiometer connected to the manipulandum provided a signal of wrist angular position and velocity. Visual feedback of hand position and task performance was provided to the monkeys. Agonistic and antagonistic EMG potentials were recorded using surface electrodes. RT and MT were measured only for the right hand. Highly stable and accurate baseline performances were established for both tasks.

\section{RESULTS}

Following unilateral injection of MPTP, hypokinesia and rigidity of the affected side (right) were quite prominent in all monkeys. Spontaneous rotation toward the side of the lesion was accelerated in a dose-dependent manner using $\mathrm{D}$-amphetamine (AMP) or reversed in direction using apomorphine (APO). APO rotation (tested monthly) appeared to be stable $( \pm 20 \%)$ for at least one year after MPTP injection in the unoperated controls. Similarly, clinical assessment of the affected limbs did not change once stable hemiparkinsonism was achieved. One month following intraventricular grafting, the cografted monkeys demonstrated decreased rotational behavior related to APO or AMP. Mean decrease in APO rotational behavior was $84 \%$ $(\mathrm{p}<0.01)$. The maximum change occurred $1-3$ months postoperatively, then stabilized for up to one year. A similar but less marked reduction was observed over the same timeframe in surgical controls (mean 52\%).

Clinically scored behavior was improved in all cografted monkeys; rigidity was reduced, and some fine hand movements returned to the affected hand. Clinical improvement started 1-4 months postoperatively, then stabilized 4-12 months postoperatively. No corresponding behavioral improvement was evident in any nonsurgical or surgical control.

Hemiparkinsonian monkeys demonstrated prolonged MT (bradykinesia, p <0.01), pro- 
longed RT/MO (hypo-kinesia, $\mathrm{p}<0.01$ ), and markedly disorganized EMG activity in the ballistic wrist task. All cografted monkeys showed improvement of MT back into the normal range 4-6 months postgrafting. EMG activity was better organized. The more severely parkinsonian monkeys demonstrated less recovery. RT improved less than MT. The non-surgical controls followed 6-12 months post-MPTP showed no recovery in prolonged RT or MT. Only one surgical control showed postsurgery improvement in RT and MT (for 3 months).

Separate control of left- and right-handed performance was developed and maintained with the two-handed behavioral paradigm. Impairment observed following unilateral administration of MPTP caused little or no change in left-hand performance. All cografted monkeys demonstrated recovery, but the more severe the lesion, the less complete the recovery. None of the control monkeys demonstrated recovery in the affected hand.

\section{SUMMARY OF RESULTS}

1. MPTP-lesioned non-human primates can serve as valuable models to study parkinsonism; when properly prepared, the MPTP hemiparkinsonian monkeys are behaviorally stable.

2. Lesions produced by grafting procedures alone are insufficient to provide significant degrees of fine motor recovery, especially when assessed by quantitative operant behavioral techniques.

3. Cografts can improve but not totally reverse parkinsonian symptoms.

4. The severity of the MPTP lesion seems important in limiting fine motor behavioral recovery.

Supported by USPHS Grant RROO165 and NINCDS N24340. 

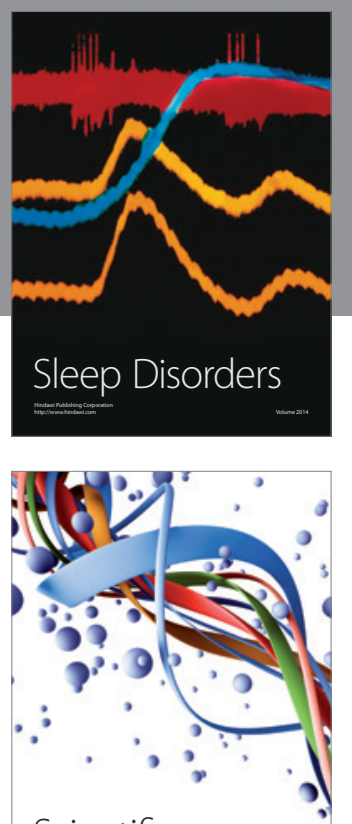

Scientifica
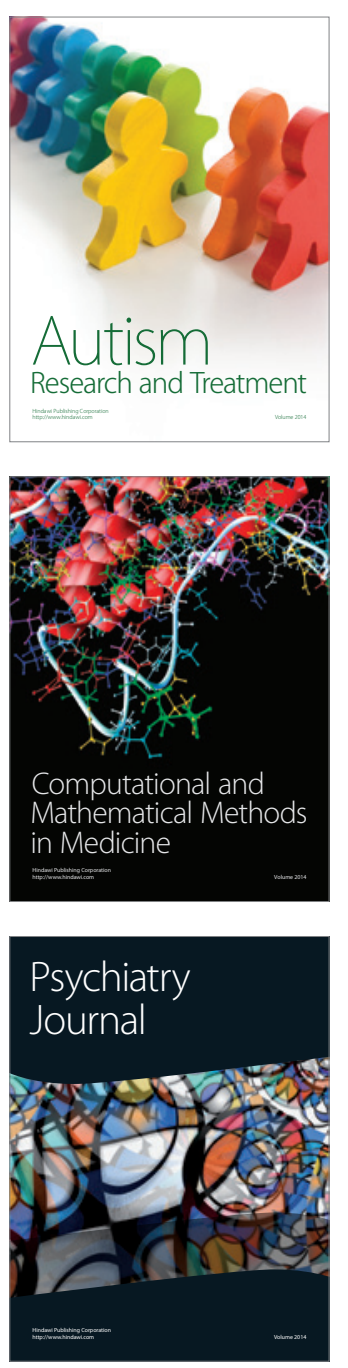
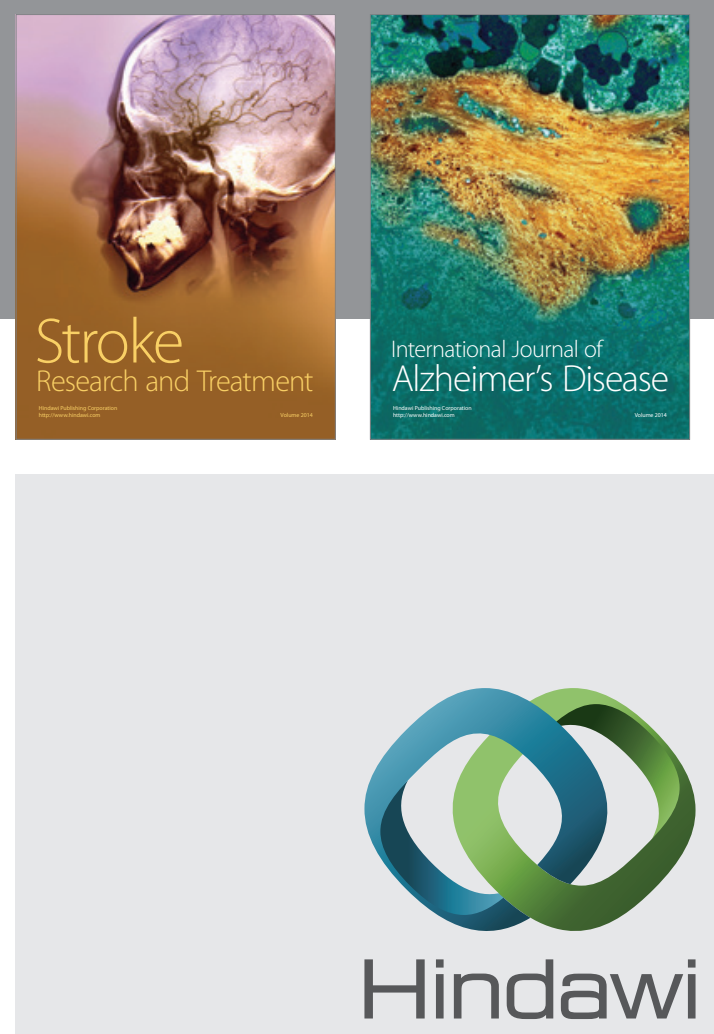

Submit your manuscripts at

http://www.hindawi.com
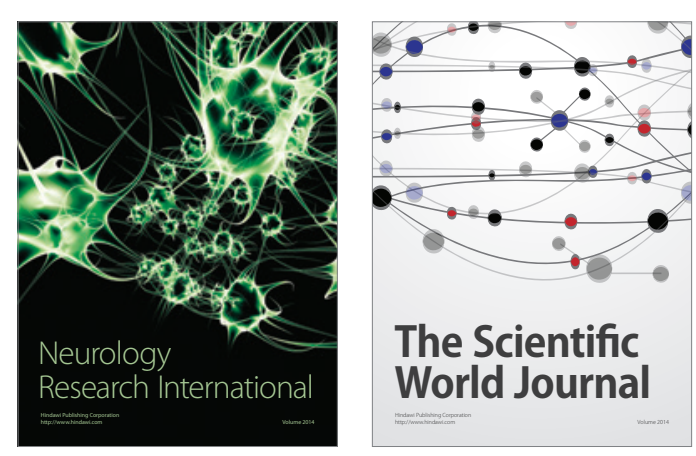

The Scientific World Journal

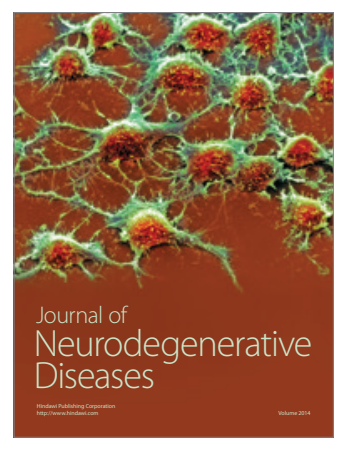

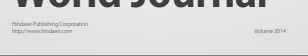

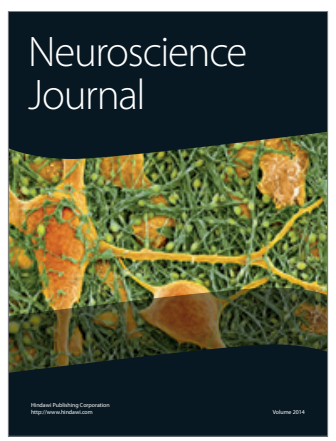

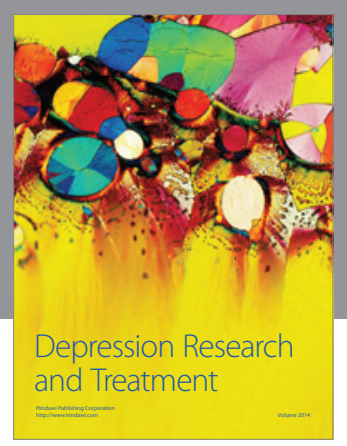
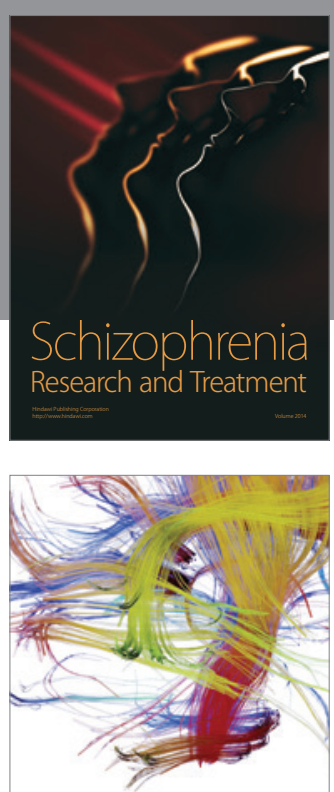

Brain Science



Neural Plasticity


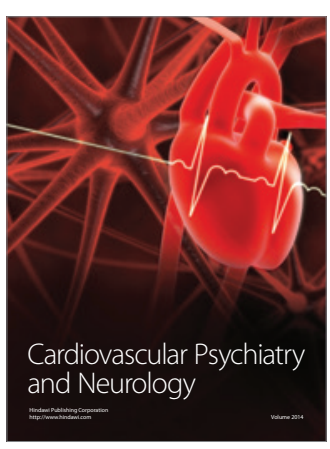

Parkinson's

Disease
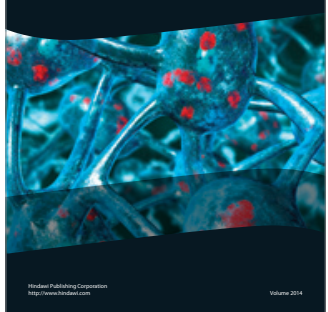